Method A retrospective cohort study of patients with CD and UC on infliximab at Milton Keynes General hospital were included in this study. Patients were identified from the infliximab database and the medical records were reviewed. The duration of infliximab therapy was recorded along with the ability to withdraw therapy at yearly review.

Results Forty-five patients were included for analysis of which $58 \%$ were male. The median age was 36.6 years. The majority of patients had a diagnosis of Crohn's disease (42/45). The mean follow up period of patients was 1.6 years (range 0-4 years). Mucosal healing was noted in $33.3 \%$ of patients. Six patients were able to stop Infliximab therapy of which $66.6 \%$ were stopped within the first two years of therapy. None of these patients required repeat infliximab therapy. There was no correlation between disease duration/age of patient or disease location in our ability to stop infliximab therapy.

Conclusion Infliximab is very successful at inducing mucosal healing and inducing clinical remission. When patients were assessed at year 1 , the vast majority were not able to stop infliximab due to poor symptom control or ongoing endoscopic disease. Significantly more patients were able to stop therapy by the second year of therapy. We conclude, that from our experience, the ideal point to consider stopping infliximab therapy is within the first two years of therapy and this correlates very well with the soon to be published European consensus recommendations (EPACT-2).

\section{WITHDRAWAL OF MAINTAINENCE INFLIXIMAB THERAPY}

L Lee, J Boyd, S Lanzon-Miller Department of Gastroenterology, Milton Keynes General Hospital, UK

\subsection{6/gutjnl-2013-305143.81}

Aims/Background Infliximab (IFX) is a chimeric monoclonal antibody effective for inducing and maintaining remission in Crohn's disease (CD) and ulcerative colitis (UC). Long term therapy is efficacious for maintaining clinical remission; however, infliximab-use is associated with significant side effects and considerable costs. NICE recommends that patients be assessed for cessation of therapy on a yearly basis. This audit analyses our ability to withdrawal infliximab for patients established on maintenance therapy. 